pervaded the legal proceedings over the difference between accreditation within the United Kingdom and specialist certification in the European Community. The joint committee had correctly advised Dr Goldstein that he had fulfilled the training requirements of the medical directives of the European Community, and could apply to the General Medical Council for the certificate of specialist training, a qualification that has no legal standing in the United Kingdom but is recognised as indicating specialist status by other states within the European Community. The judge found it "perverse" that the committee should recommend this while refusing accreditation. The two indicators, however, bear no relation to each other. ${ }^{+}$Specialists in other European countries work mainly in free practice outside hospitals, where they do not have the same responsibilities as NHS consultants. Their certificates are a legal requirement for them to be able to claim reimbursement of specialist fees through social security systems but do not indicate suitability for a senior hospital appointment.

Accreditation is a purely British phenomenon with no meaning in European Community law and is designed to fulfil a uniquely British need. This distinction is widely misunderstood but is likely to become increasingly important as more doctors migrate within the European Community. The joint committee is at fault in that the relevant guidance in its handbook is confusing.

The outcome in this case leaves many questions unanswered, but some conclusions may be drawn. Firstly, bodies acting in the public domain, and therefore subject to judicial review, should be aware of legal pitfalls, and may need to employ legal assessors to avoid them. The procedures and criteria used by the joint committee did not stand up to judicial scrutiny, and better procedures will be needed in future. Secondly, a more rigorous analysis of the criteria applied in accrediting trainees and a more cogent justification of them are needed. Considerations to do with education and manpower have become tangled, and the joint committee must now try to separate them, perhaps by introducing a numbering system for trainees similar to that used in surgery. Lastly, the case has highlighted the unsatisfactory nature of temporary senior registrar appointments. They are an inefficient way of maintaining a clinical service, encourage expectations in the appointee that may not be fulfilled, and are not subject to the same quality controls as substantive posts. In future, such posts should be made available to visiting registrars who may not otherwise be able to obtain experience in higher training before returning to their countries of origin.

STEPHEN BREARLEY

Consultant Surgeon,

Whipps Cross Hospital,

London E11 INR

1 Lister J. Accreditation: who needs it? Br f Hosp Med 1985;34:323

2 Brahams D. Medicine and the law. Specialist training and accreditation. Lancet 1991;338:1585-6. 3 General Medical Council. Recommendations on the training of specialists. London: GMC, 1987.

4 Brearley S. Medicine in Europe. Medical education. BM7 1992;304:41-4.

\title{
Any better? Outcome measures in medical audit
}

\section{Patients care about outcome; doctors should be measuring it}

Although the separation between purchasers and providers may have some desirable features, it is tending to create a gulf between the people who need information and the people who are in a position to provide it. Take managers in purchasing authorities, confused about why consultants in their patch elect for widely differing treatments for apparently similar patients. As well as knowing the different costs they would want to know whether the patients have benefited from their treatment six months or a year later. How could they find out?

They might have to rely on the skill of consultants in provider units. But why should their managers sanction this possibly expensive work? What's in it for them? It is as if conscientious plumbers visited clients' homes several months after finishing each job to discover faults in their work that no one had complained about. Perhaps the primary health care players in this game are better placed to discover some knowledge of outcome-general practice fundholders are in a good position to vote with their patients' feet and avoid individual consultants, hospitals, or even districts whose treatment proves less than adequate. Yet individual general practitioners are unlikely to have sufficient caseload or knowledge of the relative success of comparable units to make rational decisions.

Measurement of clinical outcomes is increasingly being seen as crucial for monitoring quality and achieving cost effectiveness. Politicians like it because they believe that the objective of health care is to maximise the health of the community and because it offers a way of making the views of consumers central to the provision of health services. Managers like it because they want to have a better idea of what they are paying for so that they can choose the best for the available money. Doctors like it because they want any moves towards managed care to mean providing the best and not just the cheapest.

The debate concerning the value of routinely used outcome measures as a managerial as well as a clinical tool has been fired by the prospect of a health care system that attempts to organise the provision of care according to the health needs of the population. Such a system can function only if it succeeds in finding a way of identifying and measuring health gain, lending urgency to the need for evidence to challenge the view-still sometimes held-that outcome measures other than avoidable mortality and standardised mortality ratios are illusory. Doctors need to know what benefits existing treatments bring, whether they can do it better, and indeed whether they should be doing anything at all. Until purchasers find ways of deriving this information from their contracts, the development of outcome measures will remain the pipedreams of enthusiasts.

This is not the case in the United States, where concerns over quality and variations in the delivery of care have triggered a substantial body of work by health care professionals. With increasing attempts to reduce variations in practice the need has arisen for straightforward measures that are sensitive to the effects of treatment on health. In particular, the United States medical outcome studydesigned to assess process and outcomes of care for patients with chronic disease according to variations in the style of practice in different systems of care-has made important strides in this direction.

This approach recognised the prior need to get doctors on board. Moreover, it measured outcomes of prime concern to 
patients. Initially working with four main conditionshypertension, arthritis, gastrointestinal disorders, and myocardial infarction - the study devised a generic health status questionnaire providing a yardstick for comparing different conditions. Its short general survey comprised six health concepts: three (physical, social, and role functioning) collected information on dysfunction caused by ill health, and three (mental health, perceptions of overall health, and intensity of pain) measured more subjective components of health and general wellbeing. All measures were rated on scales of $0-100$, with higher scores indicating better health. Regression coefficients estimated the effect of single and combined chronic conditions on function and wellbeing, controlling for factors such as sex, age, income, education, and other coexisting illness. The impact of each chronic condition on each health status measure was estimated as the mean deviation from a standard score for patients without chronic conditions. Results were presented graphically for average patients with each condition across each health measure.

Does this help our understanding? Reassuringly, the profiles so derived showed a startling ability to discriminate between chronically ill patients and patients with no chronic disease as well as a general population. Given the measures, the findings contribute to an understanding of the importance of chronic disease from the patient's perspective. For example, a nine point difference in physical functioning is equivalent to the effect of having arthritis or back problems. A 13 point difference in perception of health is equivalent to the effect of diabetes or congestive heart failure. Perceptions of health were poorest for patients with congestive heart failure and gastrointestinal disorders and best for patients with hypertension or back problems. Physical function was best for hypertensive patients and poorest for those with myocardial infarction or congestive heart failure. The worst role function was found in patients with myocardial infarction or congestive heart failure and the best in patients with hypertension. Pain also varied among patients, with those with arthritis scoring most pain and those with hypertension least pain. Distinct patterns emerged from the consistency of differences in health status shown by each condition. Such information increases our understanding of the needs of chronically ill patients and what should be done to meet them.

This work is a beginning. The new centres for information on outcomes and clinical effectiveness funded by the Department of Health will help to disseminate the results of these and similar efforts and stimulate further developments and refinements. Additionally, however, two important tasks need tackling by doctors and managers within the NHS before the use of outcome measures can become widespread. The first is a programme of research and development to guide the introduction of outcome measurement in the NHS, beginning perhaps by applying American results to the British experience. The second is to foster an environment in which the interests of the public are paramount. This means finding ways of improving the provision of information to patients to enable their involvement in decisions concerning their own care. The collection and use of information on health outcomes will challenge the potentially destructive competitive instincts of some health managers and the overweening preoccupation of some doctors to restrict the flow of information under the guise of protecting their clinical freedom.

To realise these objectives fully, however, requires the establishment of effective working relationships between general practitioners and hospital consultants, doctors and provider managers, and purchasers and providers. The NHS and its patients would be the winners if outcomes become central to health service management.

Public Health Specialist,

ALISON FRATER

North West Thames Regional Health Authority,

London W2 3QR

King's Fund Centre,

London NW1 $7 \mathrm{NF}$

1 Tarlov AR, Ware JE, Greenfield S, Nelson EC, Perrin E, Zubkoff $M$. The medical outcome study: an application of methods for monitoring the results of medical care. $\mathcal{F A M A} 1989 ; 262: 925-30$.

\section{Extensible bandages}

\section{Should be dispensed with more information on performance}

For many years doctors and nurses have selected their bandages without any clear knowledge of how the material will perform or what effect it will have on the limb. This happy state of ignorance was disturbed when high compression bandaging was shown to promote rapid healing of venous ulcers in most patients. ${ }^{1}$ District nurses are now asking why they cannot have access to these bandages.

Meanwhile, the Department of Health has recognised that no truly elastic bandages appropriate for sustained compression of the lower limb are available on prescription. This was the case for elastic stockings until a few years ago when a British standard classification was introduced for graduated compression hosiery, which became prescribable. It therefore seemed appropriate to follow a similar procedure, and the Surgical Material Testing Laboratory set about formulating a procedure for classifying bandages. ${ }^{2}$

For lightweight conforming stretch bandages (type 1), suitable for retaining dressings, and light support bandages (type 2) such as crepe, suitable for supporting joints and preventing oedema, the classification usefully describes the function of these bandages. It is when we come to compression bandages (type 3 ) that doctors start to get anxious. Why will clinically effective bandage regimens that have stood the test of time no longer be acceptable whereas new and virtually untried bandages are being swiftly recognised? It seems that to qualify a bandage must fit neatly into arbitrary bands of compression as defined in the testing procedure. This makes sense for elastic stockings, which are manufactured specifically to fit different sizes of leg, but with bandages we must also consider Laplace's law, which tells us that the pressure exerted by a bandage is inversely related to the diameter of the limb. A moderate compression bandage giving 18-24 $\mathrm{mm} \mathrm{Hg}$ compression to an average ankle (type $3 \mathrm{~b}$ ) might easily apply compression of twice this to the chicken like ankles of a fragile elderly woman while applying virtually no useful pressure to an ankle affected by the massive chronic oedema of untreated venous or lymphatic insufficiency. Classifying bandages into type 3a (light compression), 3b (moderate compression), $3 \mathrm{c}$ (high compression), and $3 \mathrm{~d}$ (extra high performance compression) is therefore misleading. Doctors and nurses could find themselves answering charges of negligence if pressure necrosis followed the application of a bandage that 